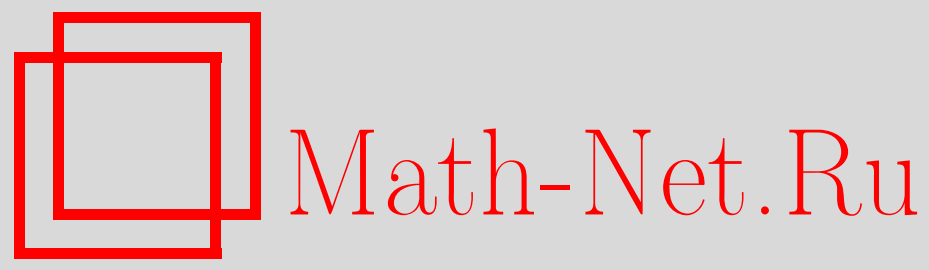

Д. А. Панов, Затягиваемые кривые и теорема Мёбиуса о трех точках перегиба, Функи. анализ и его прил., 1998, том 32, выпуск 1, 29-39

DOI: https://doi.org/10.4213/faa395

Использование Общероссийского математического портала MathNet.Ru подразумевает, что вы прочитали и согласны с пользовательским соглашением

http://www . mathnet.ru/rus/agreement

Параметры загрузки:

IP : 54.198 .64 .247

26 апреля 2023 г., 15:57:06

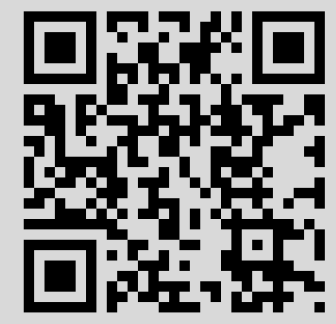


Функииональньй анализ и еәо приложения

1998, т. 32, вып. 1, с. 29-39

УДК 514

\title{
Затягиваемые кривые и теорема Мёбиуса о трех точках перегиба
}

\author{
(c) 1998. Д. А. ПАнов
}

\section{Введение}

Классическая теорема Мёбиуса утверждает, что нестягиваемая кривая, вложенная в проективную плоскость, содержит не менее трех точек перегиба [1]. Любая нестягиваемая кривая должна содержать нечетное число точек перегиба, но если кривая не является вложенной, то она может содержать лишь одну точку перегиба (рис. 1).

ЗАМЕЧАНИЕ. В настоящей работе под кривой всегда понимается замкнутая кривая, т. е. отображение из окружности в некоторую поверхность.

В работе [2] была высказана гипотеза, что любая кривая, которую можно получить из прямой деформацией, не содержащей опасных самокасаний, должна содержать не менее трех точек перегиба. Под опасными самокасаниями понимаются те, при которых ориентация кривой индуцирует на соприкасающихся ветвях одинаковые направления (рис. 2). Теорема 1 настоящей работы является частичным подтверждением этой гипотезы.

ТЕОРема 1. Пусть кривая на проективной плоскости $C^{1}$-близка к прямой и имеет не более пяти точек перегиба. Если рассматривать гомотопию кривой в классе иммерсированных кривых, не содержащих опасных самокасаний, то в результате такой гомотопии невозможно получить кривую с единственной точкой перегиба, не пройдя в прочессе гомотопии через кривую с семью точками перегиба.

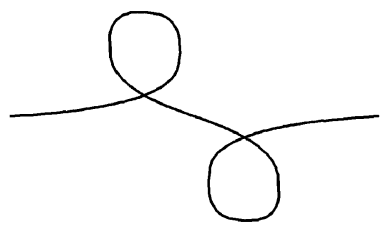

Рис. 1

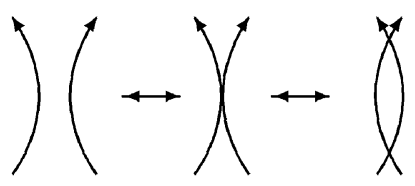

Рис. 2

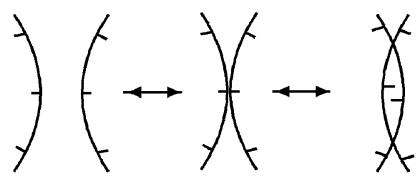

Рис. 3

Доказательство этой теоремы основывается на построении инвариантов деформаций двойственных кривых - в данном случае волновых фронтов на сфере. При этом возникают следующие понятия.

ОПРЕДЕлЕниЕ 1. Деформация кривой в классе гладко иммерсированных коориентированных кривых, лежащих на некоторой поверхности, называется

* Работа частично поддержана Российским фондом фундаментальных исследований (проект 96-01-01104). 
прокальвающей, если в процессе деформации не происходит самокасаний, при которых коориентирующие нормали обеих пересекающихся ветвей направлены внутрь образуемой ими луночки (рис. 3).

ОПРЕДЕЛЕНИЕ 2. Пусть $M$ - замкнутая двумерная ориентируемая поверхность и $\gamma$ - замкнутая связная коориентированная кривая, лежащая на $M$. Кривая $\gamma$ называется затягиваемой, если существует замкнутая поверхность $N$ с непустой границей $\partial N$, коориентированной наружу, и иммерсия $N \rightarrow M$, при которой $\partial N$ иммерсируется на $\gamma$ с сохранением коориентации.

ОПРЕДЕЛЕниЕ 3 . Пусть $\gamma: S^{1} \rightarrow S^{2}-$ некоторая гладкая кривая, образ которой снабжен коориентацией. Поляризацией для точки $x \in S^{2}$ называется гладкое отображение диска $f: D \rightarrow S^{2}$, такое, что

a) $f$ совпадает с $\gamma$ на окружности $S^{1}=\partial D$;

б) $f$ имеет единственную точку ветвления $y$ типа $z^{2}, y \notin \partial D$, причем $f(y)=x$ и $f$ является иммерсией всюду вне $y$;

в) коориентация кривой $\gamma\left(S^{1}\right)$ согласована при этой иммерсии с внешней коориентацией диска $D$.

Вложенная в плоскость коориентированная наружу окруж-

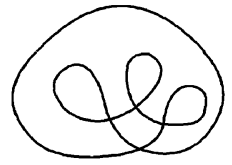

Рис. 4

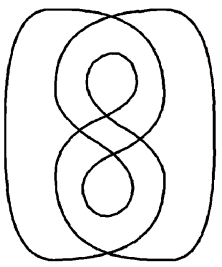

Рис. 5 ность является затягиваемой кривой. Менее тривиальные примеры затягиваемых кривых изображены на рис. 4 и 5. Первая кривая затягивается тором с выкинутым диском. Вторая кривая затягивается диском двумя неэквивалентными способами (этот пример принадлежит Дж. Милнору) [3]. Эти способы не переводятся друг в друга никакими деформациями затягивающих отображений, при которых $\partial N$ иммерсируется на $\gamma$.

Для каждой кривой можно определить серию чисел $I(g, d)$ - чисел попарно неэквивалентных затягиваний сферой с $g$ ручками и $d$ выкинутыми дисками.

Теорема 2. Свойство кривой быть затягиваемой инвариантно относительно прокальвающей деформачии. Все иисла $I(g, d)$ также являются инвариантами.

Этот факт очевиден, если в процессе деформации не происходит самокасаний и прохождений через тройные точки, и непосредственно проверяется, если они происходят.

В $\S 1$, основываясь на понятиях из определений 1,2 и на теореме 2 , мы доказываем усеченный вариант теоремы 1:

ТЕОРема 1'. Пусть кривая на проективной плоскости $C^{1}$-близка к прямой и имеет не более трех точек перегиба. Тогда при помощи гомотопии кривой в классе иммерсированных кривых, не содержаших опасных самокасаний, ее нельзя перевести в кривую с единственной точкой перегиба, не пройдя в прочессе гомотопии через кривую с пятью точками перегиба.

В $\S 2$ изучаются свойства, которым удовлетворяют поляризации кривых. В $\S 3$ каждой кривой сопоставляется некоторый геометрический объект (являющийся двумерным многообразием с краем и углами); он служит инвариантом прокалывающих деформаций (теорема 3 ). Наконец, в $\S 4$ дается полное доказательство теоремы 1 , основанное одновременно на технике затягиваемых и поляризуемых кривых. 


\section{§1. Доказательство усеченной теоремы}

Множество ориентированных прямых на проективной плоскости образует сферу $S^{2}$. Фронтом, двойственным проективной кривой, называется сферическая кривая, образованная ориентированными касательными исходной.

ОПРЕДЕлЕНИЕ 4. Фронт, двойственный кривой, гладко иммерсированной в проективную плоскость, называется звездным фронтом.

Звездный фронт может иметь особенности. Его типичными особенностями являются двойные точки (соответствующие бикасательным исходной кривой) и обычные точки возврата (соответствующие точкам перегиба исходной кривой). На звездном фронте отсутствуют точки перегиба, так как они соответствовали бы точкам возврата исходной кривой.

Селаживанием звездного фронта называется кривая, полученная из фронта сглаживанием точек возврата (рис. 6). Сглаженный звездный фронт обладает естественной коориентацией. Вне точек сглаживания эта коориентация задается направлением в

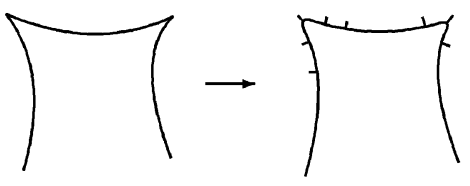

Рис. 6 сторону, противоположную выпуклости фронта. Из рис. 6 видно, что задающаяся так коориентация продолжается и на окрестности точек сглаживания.

Рассмотрим деформацию иммерсированных кривых в классе кривых с фиксированным числом точек перегиба. Эта деформация задает деформацию соответствующих двойственных звездных фронтов и их сглаживаний (путем согласованного сглаживания фронтов в процессе деформации).

Лемма 1. Если при деформации исходной кривой не происходит опасных самокасаний, то деформачия сглаживания двойственного фронта является прокальвающей.

СлЕДСТВИЕ. Если в условиях леммы сحлаживание фронта начальной кривой является затягиваемым, то таковым является и сдлаживание фронта конечной кривой.

ДокаЗАТЕЛЬСТво ЛЕммы 1. Рассмотрим общую деформацию проективной кривой, не меняющую числа точек перегиба. Деформация двойственного фронта будет содержать лишь следующие элементарные перестройки: прохождения тройных точек (рис. 7) и прокалывания (рис. 8). При этом самокасания фронта исключены, так как они соответствовали бы опасным самокасаниям двойственной кривой, исключенным по определению. Поэтому са-
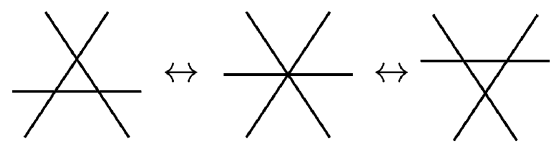

Рис. 7

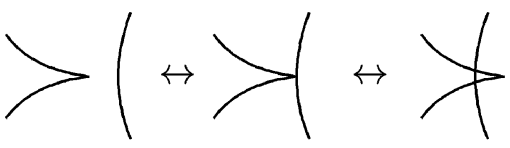

Рис. 8 мокасания сглаженных фронтов могут соответствовать исключительно прокалываниям самих фронтов. Легко показать, что среди этих самокасаний отсутствуют запрещенные в определении 1. Лемма доказана.

ЗАмЕчАниЕ. Если разрешить кривой менять число точек перегиба в процессе деформации, то к элементарным перестройкам двойственных фронтов 
добавится еще одна перестройка - рождение или смерть пары точек возврата (рис. 9).

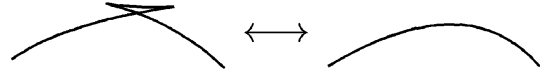

Рис. 9

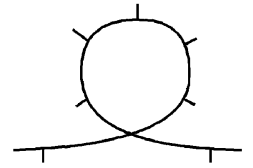

Рис. 10

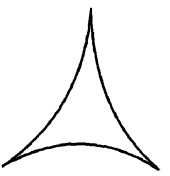

Рис. 11

Следующее геометрически очевидное утверждение доказано в [4].

ЛЕмма 2. На затягиваемой кривой не может суиествовать изолированной коориентированной наружу петли, т. е. фрагмента, изображенного на рuс. 10.

ДОКАЗАТЕЛЬСтво теОРЕмЫ $1^{\prime}$. Рассмотрим кривую $\gamma$ на проективной плоскости, $C^{1}$-близкую к прямой и имеющую три точки перегиба. Фронт, двойственный такой кривой, имеет вид, как на рис. 11 (это проверяется непосредственным вычислением). Сглаживание такого фронта является затягиваемой кривой. Рассмотрим произвольную гомотопию кривой $\gamma$ в классе гладко иммерсированных кривых. Из следствия леммы 1 вытекает, что до тех пор, пока на деформируемой кривой $\gamma_{t}$ будет три точки перегиба, сглаживание двойственного фронта $f_{t}$ будет затягиваемой кривой. По лемме 2 на затягиваемой кривой не может быть петли, коориентированной наружу. Значит, на фронте $f_{t}$ не может присутствовать непосредственно сокращающейся пары точек возврата (рис. 9). Последнее означает, что на кривой $\gamma_{t}$ никогда нельзя сократить пару точек перегиба. Теорема доказана.

\section{§2. Поляризация и ее свойства}

Начиная с этого параграфа, мы будем рассматривать лишь коориентированные кривые и волновые фронты, лежащие на сфере.

ОПРЕДЕЛЕНИЕ 5 . Пусть $x$ и $y$ - некоторые точки на сфере, соединенные путем $\rho:[0,1] \rightarrow S^{2}, \rho(0)=x, \rho(1)=y$. Будем говорить, что вдоль пути $\rho$ задана гладкая поляризаиия, если поляризация задана для каждой точки пути $\rho$ и гладко зависит от точки. Продолжить поляризацию из $x$ в $y$ вдоль пути $\rho$ - это значит задать гладкую поляризацию вдоль $\rho$, совпадающую с начальной поляризацией в $x$.

Следующие рассуждения вместе с леммой 3 определяют те допустимые пути, вдоль которых поляризацию всегда можно продолжить.

Пусть $x$ - некоторая точка сферы, заданная вместе с ее поляризацией $f: D \rightarrow S^{2}$. Полный прообраз точки $x$ под действием поляризующего отображения $f$ обозначим через $\left\{z, z_{1}, \ldots, z_{n}\right\}=f^{-1}(x)$, где $z$ - единственная точка ветвления. Рассмотрим произвольный путь $\rho$ на сфере, соединяющий точку $x$ с некоторой точкой $y, \rho:[0,1] \rightarrow S^{2}, \rho(0)=x, \rho(1)=y$.

ОПРЕДЕЛЕНИЕ 6. Будем называть путь $\rho$ доnустимыл, если на диске $D$ существует гладкий путь $\tilde{\rho}$, проходящий через точку ветвления $z$ и двулистно накрывающий путь $\rho$, т. е. $\tilde{\rho}:[-1,1] \rightarrow D, f(\tilde{\rho}( \pm t))=\rho(t), \tilde{\rho}(t) \neq \tilde{\rho}(-t)$ (рис. 12).

ЛЕмма 3. Поляризаиию из точки $x$ можно продолжить вдоль пути $\rho$, если путь $\rho$ является допустимьм. 
ДокАЗАТЕЛЬСтво. Пусть $\Omega_{\varepsilon}$ есть $\varepsilon$-окрестность пути $\rho([0,1])$. Рассмотрим прообраз этой области под действием поляризующего отображения $f$. Обозначим через $\widetilde{\Omega}_{\varepsilon}$ ту из компонент связности прообраза области $\Omega_{\varepsilon}$, которая содержит путь, двулистно накрывающий путь $\rho([0,1])$. Область $\widetilde{\Omega}_{\varepsilon}$ будет двулистно накрывать область $\Omega_{\varepsilon}$ (рис. 13).

Поляризацию вдоль пути $\rho$ будем строить, деформируя отображение $f$. Мы будем изменять это отображение только для точек области $\widetilde{\Omega}_{\varepsilon}$. Рассмотрим однопараметрическое семейство $F_{t}$ автоморфизмов (взаимно однозначных диффеоморфизмов на себя) области $\Omega_{\varepsilon}$, постоянных вдоль границы области и переводящих точку $x$ в точку $\rho(t): F_{t}(x)=\rho(t)$. Это семейство автоморфизмов области $\Omega_{\varepsilon}$ породит семейство отображений $\tilde{f}_{t}$ из диска $D$ в сферу: $f_{t}(y)=$ $F_{t}(f(y))$, если $y \in \widetilde{\Omega}_{\varepsilon}, f_{t}(y)=f(y)$, если $y \notin \widetilde{\Omega}_{\varepsilon}$. Семейство отображений $\tilde{f}_{t}$ доставит поляризацию вдоль пути $\rho$. Лемма доказана.

СЛЕДСТВИЕ. Поляризачию можно продолжить вдоль любого пути, не пересекающего кривой $\gamma$.

Рассмотрим множество областей, на которые кривая $\gamma$ разбивает сферу. Будем называть эти области элементарными. Из леммы 3 вытекает, что, задав поляризацию во внутренней точке одной из элементар-

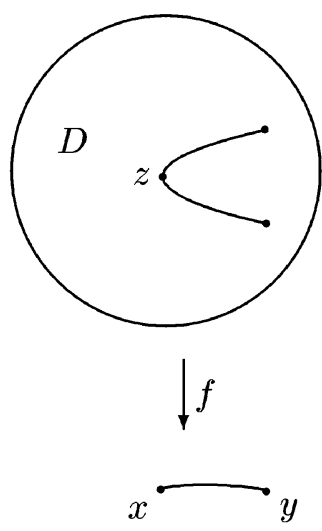

Рис. 12
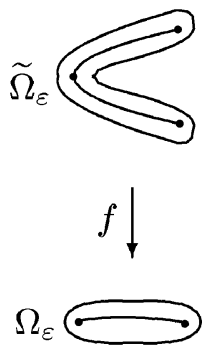

Рис. 13 ных областей, мы можем гладко продолжить поляризацию на всю область. Из односвязности элементарных областей следует, что продолжение поляризации не зависит от выбора пути. Элементарную область с выбранной гладким образом поляризацией будем называть поляризованной областью.

Следующие две леммы говорят о том, каким образом поляризация продолжается из одной элементарной области в соседние элементарные области.

ЛЕмма 4. Пусть $\Omega_{1}$ и $\Omega_{2}$ - две соседние элементарные области, граничащие по некоторому отрезку $I$ и коориентация на I направлена внутрь области $\Omega_{2}$. Тогда всякая поляризачия области $\Omega_{2}$ продолжается внутрь области $\Omega_{1}$.

ДокАЗАТЕльство. Пусть $x$ - некоторая точка области $\Omega_{2}$, заданная вместе с поляризацией $f: D \rightarrow S^{2}$. Рассмотрим путь $\rho([0,1])$, содержащийся внутри $\Omega_{2}$ и соединяющий точку $x$ с произвольной точкой отрезка $I$. Рассмотрим путь $\tilde{\rho}$, двулистно накрывающий путь $\rho$. Докажем, что путь $\tilde{\rho}$ не может пересекать границы диска $D$. Предположим противное, т. е. что точка $\tilde{\rho}(1)$ или $\tilde{\rho}(-1)$ лежит на границе диска $D$. Если $\tilde{\rho}(1) \in \partial D$, то отрезок $f(\tilde{\rho}([1,1-\varepsilon]))$ должен быть направлен в сторону, обратную коориентации отрезка I. Это означает, что точка $f(\tilde{\rho}(1-\varepsilon))$ лежит в области $\Omega_{1}$, чего 


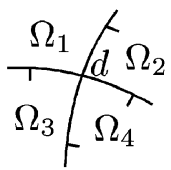

Рис. 14

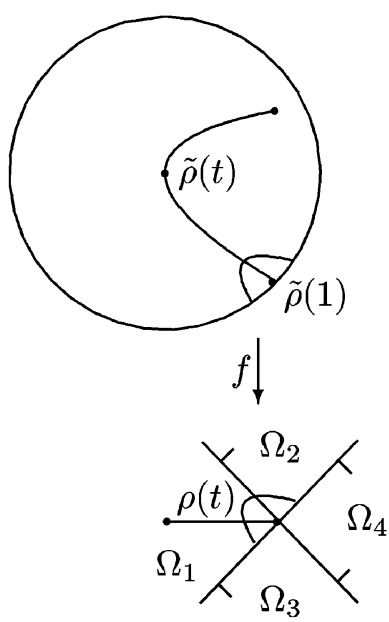

Рис. 15

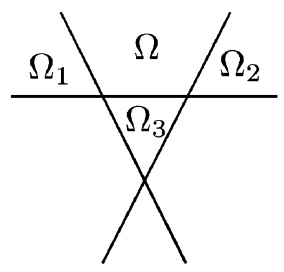

Рис. 16

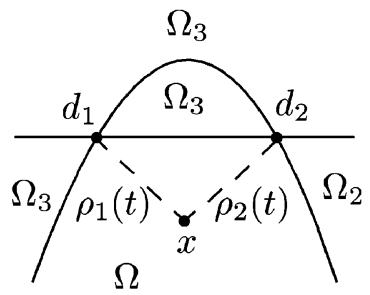

Рис. 17 не может быть. Поэтому путь $\tilde{\rho}$ лежит целиком во внутренности диска $D$. А значит, путь $\rho$ можно так удлинить, чтобы его конец попал в область $\Omega_{1}$ и в то же время удлиненный путь оставался допустимым путем. По лемме 3 последнее означает, что поляризацию из точки $x$ можно продолжить в некоторую точку области $\Omega_{1}$, а следовательно, во всю область. Лемма доказана.

Лемма 5. Пусть $d$ - некоторая двойная точка кривой $\gamma$ и $\Omega_{1}, \Omega_{2}, \Omega_{3}, \Omega_{4}$ - элементарные области, прилегающие $\kappa d$, причем коориентачия направлена из $\Omega_{1}$ в $\Omega_{2}$, из $\Omega_{1}$ в $\Omega_{3}$, из $\Omega_{2}$ в $\Omega_{4}$ и из $\Omega_{3}$ в $\Omega_{4}$ (рис. 14). Тогда верно следуюшее:

1) поляризачию из области $\Omega_{4}$ можно продолжить во все три области $\Omega_{1}, \Omega_{2}, \Omega_{3}$;

2) поляризацию из $\Omega_{2}$ или $\Omega_{3}$ всегда можно продолжить в $\Omega_{1}$;

3) если поляризачия из $\Omega_{1}$ продолжается в $\Omega_{2}$ $u \Omega_{3}$, то она продолжается $и$ в $\Omega_{4}$.

ДоказАтельство. Утверждения 1) и 2) автоматически вытекают из леммы 4. Докажем утверждение 3$)$. Пусть $x$ - произвольная точка области $\Omega_{1}$ с фиксированной поляризацией. Рассмотрим путь $\rho$, соединяющий точку $x$ с точкой $d$, и возьмем на диске $D$ путь $\tilde{\rho}$, двулистно накрывающий $\rho$. Докажем от противного, что концы пути $\tilde{\rho}$ не лежат на крае диска.

Предположим, что $\tilde{\rho}(1) \in \partial(D)$. Рассмотрим маленький полудиск с центром в точке $\tilde{\rho}(1)$ (рис. 15$)$. Без ограничения общности можно считать, что под действием отображения $f$ этот полудиск переходит в области $\Omega_{1}$ и $\Omega_{2}$. Сразу же видно, что в такой ситуации невозможно продолжить поляризацию из точки $x$ в область $\Omega_{3}$. Противоречие.

Таким образом, путь $\tilde{\rho}$ целиком лежит внутри диска $D$, а значит, путь $\rho$ можно так продлить в область $\Omega_{4}$, чтобы продленный путь оставался допустимым. Лемма доказана.

Следующие две леммы понадобятся при доказательстве теоремы 3 .

Лемма 6. Пусть кривая $\gamma$ имеет треугольный фрагмент вида, изображенного на рис. 16. Поляризачия из области $\Omega$ должна продолжаться хотя би в одну из областей $\Omega_{1}, \Omega_{2}$ или $\Omega_{3}$.

ЛЕмма 7. Пусть кривая $\gamma$ имеет фрагмент вида, изображенного на рис. 17. Если поляризачия, определеннал в области $\Omega$, продолжаетсл хотл бы в одну из областей $\Omega_{1}$ или $\Omega_{2}$, то она продолжаетсл и в обе области. 
ДоКАЗАТЕЛЬСТВо ЛЕММЫ 6 . Пусть $x$ - некоторая точка из области $\Omega$, заданная вместе с поляризацией. Предположим от противного, что поляризация не продолжается ни в одну из областей $\Omega_{1}, \Omega_{2}, \Omega_{3}$. Из этого следует, что коориентация на границе области $\Omega$ направлена во внешность. Рассмотрим два пути $\rho_{1}, \rho_{2}$, соединяющие точку $x$ с двойными точками $d_{1}$ и $d_{2}$.

Пусть пути $\tilde{\rho}_{1}, \tilde{\rho}_{2}$ двулистно накрывают пути $\rho_{1}, \rho_{2}$. Все концы путей $\tilde{\rho}_{1}, \tilde{\rho}_{2}$ обязаны лежать на границе диска $D$ (иначе поляризацию можно было бы продолжить хотя бы в одну из областей $\Omega_{1}, \Omega_{2}$ или $\left.\Omega_{3}\right)$. Эти пути разобьют диск на четыре треугольных фрагмента (рис. 18). Поляризующее отображение диска индуцирует накрывающее отображение в сферу для каждого из фрагментов. В частности, один из этих треугольных фрагментов (обозначим его через $T_{1}$ ) должен взаимно однозначно отобразиться на треугольник плоскости, ограниченный путями $\rho_{1}, \rho_{2}$ и фрагментом кривой $\left[d_{1}, d_{2}\right]$. Отсюда можно извлечь, что треугольный фрагмент $T_{2}$, противоположный $T_{1}$, должен накрывать четырехугольную область с вершинами $x, d_{1}, d_{3}, d_{2}$. Но тогда граница области $T_{2}$ отображается в кривую, имеющую изолированную петлю, коориентированную наружу, что противоречит тому, что отображение накрывающее (рис. 19) (лемма 2). Лемма доказана.

ДокАЗАТЕЛЬСтво ЛЕммы 7. Пусть $x-$ некоторая точка из области $\Omega$, заданная вместе с поляризацией (рис. 17). Предположим, что поляризация из $x$ не продолжается в область $\Omega_{1}$, и выведем из этого, что поляризация не продолжается и в область $\Omega_{2}$. Рассмотрим два пути $\rho_{1}$, $\rho_{2}$, соединяющие точку $x$ с двойными точками $d_{1}$ и $d_{2}$. Пусть пути $\tilde{\rho}_{1}, \tilde{\rho}_{2}$ двулистно накрывают пути $\rho_{1}, \rho_{2}$. Рассмотрим кусок $\left[\tilde{d}_{1}, \tilde{d}_{2}\right]$ границы диска $D$, который проектируется в общую границу областей $\Omega_{3}$ и $\Omega_{4}$. Из того что поляризация не продолжается из точки $x$ в область $\Omega_{1}$, вытекает, что точка $\tilde{d}_{1}$ является одним из концов пути $\tilde{\rho}_{1}$. Отсюда легко вывести, что точка $\tilde{d}_{2}$ является одним из концов пути $\tilde{\rho}_{2}$. Поэтому поляризацию из точки $x$ нельзя продолжить и в область $\Omega_{2}$. Лемма доказана.

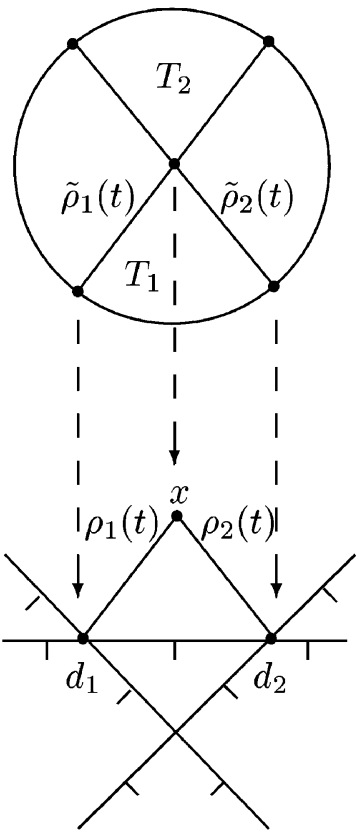

Рис. 18
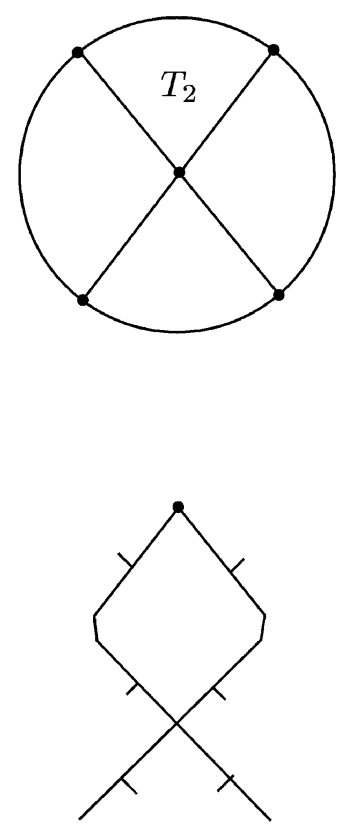

Рис. 19 


\section{§3. Поляризованная накрывающая и инварианты прокалывающих деформаций}

Сопоставим теперь всякой коориентированной кривой $\gamma$ некоторое двумерное многообразие с краем (и углами), которое мы будем называть поляризованной накрывающей.

Конструкция. Рассмотрим множество всех поляризованных элементарных областей, соответствующих кривой $\gamma$. Склеим все пары поляризованных областей, имеющих общую границу и таких, что поляризация из одной области продолжается в другую.

На рис. 20 изображены кривая и ее поляризованная накрывающая.
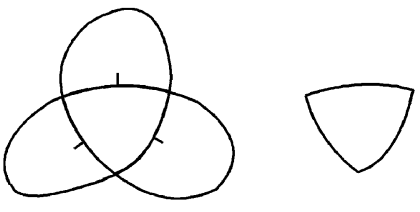

Рис. 20

Будем называть многоугольником двумерную область, каждая точка которой имеет окрестность, диффеоморфную плоскости, полуплоскости или квадранту.

ЛЕмма 8. Каждал компонента свлзности поляризованной накрывающей является многоугольником.

Эта лемма автоматически вытекает из леммы 5.

Все определения и леммы из $§ 3$ можно формулировать не только для гладких кривых, но и для фронтов, двойственных к гладким кривым. Существенным моментом является то, что для сглаживания фронта, двойственного гладкой кривой, можно определить коориентацию. Отметим, что граница поляризованной накрывающей для волнового фронта будет иметь точки возврата.

Пусть $\Phi-$ некоторый фронт на сфере, двойственный гладкой кривой проективной плоскости. Рассмотрим его поляризованную накрывающую. Всякой компоненте связности накрывающей поставим в соответствие число $2 U+V$, где $U$ - число углов компоненты, $V$ - число точек возврата компоненты. Это число назовем характеристикой компоненты связности.

ТЕорема 3. Пусть задана прокальвающая деформачия некоторого звездного фронта (допустимые перестройки - прокальвания и прохождения тройных точек). Эта деформачия порождает деформачию соответствующих поляризованных накрывающих. Тогда

1) если в прочессе деформачии фронта не происходит перестроек, то все компоненты связности поляризованной накрывающей сохраняются с точностью до диффеоморфизма (остается неизменным число углов и точек возврата на каждой компоненте связности);

2) при прохождении тройной точки все компоненты, характеристика которых отлична от 3 , сохранлютсл с точностью до диффеоморфизма; при этом могут появляться или исчезать (флуктуировать) компоненть характеристики 3 ;

3) при прокальввании все компоненты, характеристика которых отлична от 4, сохраняют свою характеристику (они не Флуктуируют, но число их углов может меняться); компоненты, имеющие характеристику 4, могут флуктуировать. 
Для доказательства этой теоремы нам понадобится следующая лемма.

Лемма 9. Пусть ү-звездный фронт. Допустим, что для некоторой точки $x$ существует поляризачия относительно фронта $\gamma$. Рассмотрим такую прокальваюшую деформацию фронта $\gamma$, в прочессе которой деформируемьй фронт не проходит через точку $x$. Тогда дяя всего семейства фронтов точка $x$ остается поляризуемой.

ДокАЗАтельство. Рассмотрим маленькую окружность с центром в точке $x$, которая не пересекается с деформируемым фронтом. Существует затягивающее отображение из цилиндра на часть плоскости, которое переводит одну часть границы цилиндра во фронт, а другую - в маленькую окружность (это отображение индуцируется поляризацией). Так как деформация фронта (объединенного с маленькой окружностью) является прокалывающей, из леммы 1 мы получаем, что объединение фронта с маленькой окружностью будет оставаться затягиваемой кривой в процессе деформации, а значит, в каждый момент деформации точка $x$ будет поляризуемой. Лемма доказана.

Доказательство утверждения 1) теоремы 3 очевидно. Докажем утверждение 2).

Рассмотрим прокалывающую деформацию звездного фронта $\gamma_{t}$, состоящую из прохождения тройной точки (рис. 7$)$. Пусть $\mu\left(\gamma_{0}\right)$ - некоторая компонента связности поляризованной накрывающей фронта $\gamma_{0}$, имеющая характеристику больше трех. Сопоставим компоненте $\mu\left(\gamma_{0}\right)$ компоненту связности поляризованной накрывающей фронта $\gamma_{1}$. Так как характеристика $\mu\left(\gamma_{0}\right)$ больше трех, естественная проекция $\mu\left(\gamma_{0}\right)$ не может целиком содержаться в треугольнике $\Delta$. Возьмем какую-нибудь поляризованную точку $x$, соответствующую многоугольнику $\mu\left(\gamma_{0}\right)$ и лежащую в области, смежной с треугольником $\Delta$. Во время деформации точка $x$ не будет задеваться фронтами $\gamma_{t}$; поэтому для каждого фронта деформации точка $x$ будет поляризуемой (лемма 9), а значит, многоугольнику $\mu\left(\gamma_{0}\right)$ мы можем поставить в соответствие некоторый многоугольник $\mu\left(\gamma_{1}\right)$.

Рассмотрим элементарные поляризованные области, из которых склеиваются многоугольники $\mu\left(\gamma_{0}\right)$ и $\mu\left(\gamma_{1}\right)$. Очевидно, что наборы должны совпадать целиком, за исключением того, что треугольная область может входить в наборы в разном количестве. Поэтому достаточно изучить, что происходит с многоугольниками рядом с треугольником $\Delta$.

Рассмотрим естественную проекцию многоугольника $\mu\left(\gamma_{0}\right)$ на сферу. Если граница многоугольника не проектируется в окрестность треугольника $\Delta$, то с $\mu\left(\gamma_{0}\right)$ в процессе перестройки ничего не происходит. Если же граница $\mu\left(\gamma_{0}\right)$ проектируется в окрестность треугольника $\Delta$, то по лемме 6 граница может проектироваться лишь в две ветви кривой, ограничивающей треугольник $\Delta$, а третья ветвь никак не будет взаимодействовать с многоугольником. Отсюда легко извлечь, что число углов многоугольника не изменится в процессе перестройки. Утверждение 2) доказано.

Доказательство утверждения 3) мы здесь не приводим, оно аналогично доказательству утверждения 2), но при этом роль леммы 6 должна играть 


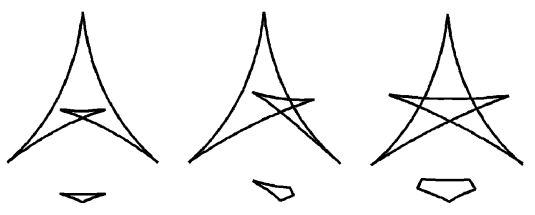

Рис. 21

лемма 7. В качестве иллюстрации к утверждению на 3) рис. 21 изображены фронты, двойственные вложенной нестягиваемой проективной кривой. Все они имеют пять точек возврата, их поляризованные накрывающие связны и имеют характеристику 5.

\section{§4. Доказательство теоремы 1}

ЛЕмма 10. Пусть $\gamma$ - кривая на проективной плоскости, $C^{1}$-близкал к прямой и имеющая не более пяти точек возврата. Тогда звездный фронт, двойственный кривой $\gamma$, может быть устроен ровно четырьмя способами с точностью до объемлющего диффеоморфизма сферы (рис. 11, рис. 21).

Эта лемма доказывается прямыми вычислениями.

Из рис. 21 видно, что если начальная кривая имеет пять точек возврата, то двойственный фронт является поляризуемым, причем поляризованная накрывающая является многоугольником характеристики 5, а если начальная кривая имеет три точки возврата, двойственный фронт является затягиваемым. Идея доказательства теоремы 1 состоит в том, что описанная ситуация имеет место не только для начальной кривой, но и в любой момент деформации кривой.

ЛЕмма 11. Рассмотрим произвольную затягиваемую кривую (звездный волновой фронт) и произведем с этой кривой перестройку рождения ласточкиного хвоста (рис. 9). При такой перестройке кривал превратится из затягиваемой в поляризуемую, причем поляризованная накрывающая полученной кривой будет содержать компоненту связности характеристики 5 .

ЛЕмма 12. Если поляризуемый фронт содержит непосредственно сокращающийся ласточкин хвост (рис. 9), то поляризованнал накрывающал фронта содержит компоненту связности характеристики 5, проектирующуюся на область, ограниченную ласточкиным хвостом.

ДоКАЗАТЕЛЬСТВо ТЕОРЕМЫ 1. В процессе деформации кривая теоретически может находиться в трех состояниях. Первое состояние - с одной точкой перегиба, второе - с тремя точками перегиба, третье - с пятью точками перегиба. Деформация начинается с кривой, находящейся во втором или в третьем состоянии. Переход из одного состояния в другое происходит за счет рождения или смерти пары точек возврата. Наша задача состоит в том, чтобы доказать, что кривая никогда на попадет в первое состояние (если деформация была без опасных самокасаний).

Теорема $1^{\prime}$ утверждает, что из состояния 2 нельзя попасть в состояние 1 , не побывав до этого в состоянии 3 , т. е. путь

$$
\text { состояние } 2 \rightarrow \text { состояние } 1
$$

невозможен. Покажем, что невозможен и следующий путь:

$$
\text { состояние } 2 \rightarrow \text { состояние } 3 \rightarrow \text { состояние } 2 \rightarrow \text { состояние } 1 \text {. }
$$


Когда мы стартуем из состояния 2, двойственный фронт является затягиваемой кривой. До того момента, когда произойдет переход в состояние 3 , двойственный фронт будет оставаться затягиваемым (теорема 2). При переходе из состояния 2 в 3 на двойственном фронте образуется ласточкин хвост, фронт станет поляризуемым и поляризованная накрывающая перестроенного фронта будет содержать компоненту связности характеристики 5 (лемма 11). Далее, до того момента, когда кривая опять попадет в состояние 2, двойственный фронт будет претерпевать прокалывающую деформацию и поляризованная накрывающая фронта постоянно будет содержать ровно одну компоненту характеристики 5 (теорема 3 ). При обратном переходе из состояния 3 в состояние 2 двойственный фронт станет затягиваемым (лемма 12). А на затягиваемом фронте нельзя сократить пары точек возврата. Утверждение доказано.

Для полного доказательства необходимо доказать, что не существует путей вида

$$
2 \rightarrow 3 \rightarrow 2 \rightarrow 3 \rightarrow \cdots \rightarrow 2 \rightarrow 3 \rightarrow 2 \rightarrow 1 \text {. }
$$

Но все такие пути содержат фрагмент $2 \rightarrow 3 \rightarrow 2 \rightarrow 1$, невозможность которого уже доказана. Теорема доказана.

\section{Заключение}

Следующая гипотеза обобщает описанные методы и из ее справедливости вытекает, что всякая кривая на проективной плоскости, которую можно получить из прямой гомотопией без опасных самокасаний, содержит не менее трех точек перегиба.

ГИПотеЗА. Пусть $\gamma$ - кривая на проективной плоскости $c 3+2 k$ точками перегиба, соединяемая с прямой деформацией без опасных самокасаний.

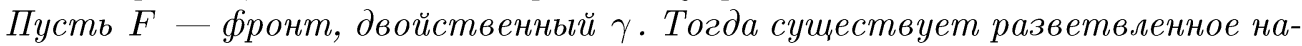
крытие области сферы диском, имеющее $k$ точек ветвления индекса 2 u гомеоморфно отображающее границу диска в фронт $F$.

Выражаю признательность В. И. Арнольду за постановку задачи и полезные обсуждения.

\section{ЛИТЕРАТУРА}

1. Sasaki $S$. The minimum number of points of inflection of closed curves in the projectiv plane. Tohoku Math. J., 9. No. 2, 113-117 (1957).

2. Арнольд В. И. Геометрия сферических кривых и алгебра кватернионов. УМН, 50, вып. 1 (301) (1995).

3. Арнольд В. И., Васильев В. А., Горюнов В. В., Ляшко О. В. Особенности. I, Современные проблемы математики. Фундаментальные направления. Т. 6 , ВИНИТИ, М., 1985.

4. Umehara $M$. 6-Vertex theorem for closed planar curve which bounds an immersed surface with non-zero genus. Nagoya Math. J., 134, 75-89 (1994). 